\title{
Rediseño Curricular de la Carrera Gestión Social y Desarrollo de la Universidad de Otavalo para su Proceso de Acreditación
}

\author{
Ledys Hernández-Chacón \\ Universidad de Otavalo, Carrera de Desarrollo Social y Cultural, Campus Cdla Imbaya, \\ Av. de los Sarances s/n y Pendoneros, Código postal: 100202, Otavalo, Ecuador. \\ (e-mail: Ihernandez@uotavalo.edu.ec)
}

Recibido Dic. 7, 2016; Aceptado Feb. 9, 2017; Versión final Abr. 12, 2017, Publicado Dic. 2017

\begin{abstract}
Resumen
El presente trabajo expone el proceso de rediseño curricular de la oferta académica en Gestión Social y Desarrollo, llevado a cabo por el equipo docente y multidisciplinar de la Universidad de Otavalo durante el año 2016. La metodología de trabajo consistió en la realización de talleres multidisciplinares, permitiendo hacer un análisis integral de los componentes del rediseño. El resultado obtenido fue una propuesta curricular armónica entre los diferentes componentes de la formación profesional, de acuerdo al modelo de competencias, que incluye las estrategias de evaluación y aseguramiento de la calidad en dicha formación. El artículo expone el curso de la transformación que se está viviendo en Ecuador con respecto a la educación superior de acuerdo al currículo por competencias. Por lo tanto este trabajo puede servir como referente a otras universidades que atraviesan el proceso de aprobación y acreditación de su oferta académica.
\end{abstract}

Palabras clave: rediseño curricular; gestión social desarrollo; educación superior

\section{Curricular Redesign of the Career Social Management and Development of the University of Otavalo for its Accreditation Process}

\begin{abstract}
This paper presents the curricular redesign of the academic offer in Social Management and Development, carried out by the teaching and multidisciplinary team of the University of Otavalo during the year 2016. The methodology consisted of the organization of multidisciplinary workshops, which allowed comprehensive analysis of the components for the redesign. The result was a harmonious curricular proposal between the different components of vocational training, according to the competency model, which includes strategies for evaluation and quality assurance in the training. The article describes the progress of the transformation that is experimenting Ecuador with respect to higher education according to the curriculum by competences. Therefore, this work can serve as a reference to other universities that go through the process of approval and accreditation of its academic offer.
\end{abstract}

Keywords: curriculum redesign; social management development; higher education 


\section{INTRODUCCIÓN}

La educación superior en Ecuador ha experimentado cambios radicales en los últimos años. Con la incorporación en la Constitución del 2008 de la educación como interés público, con derecho exclusivo del Estado a su legislación, control y regulación, el gobierno ecuatoriano desplegó una serie de normativas y políticas públicas que han marcado un rumbo decisivo para las universidades. En el año 2012 se inició un proceso evaluativo sobre la calidad y aseguramiento de la Educación Superior trayendo consigo la categorización las universidades del país. De igual manera, la oferta académica es sistemáticamente revisada a través de los procesos de acreditación y rediseño curricular. Esta transformación es una realidad en la Educación Superior latinoamericana. Desde la década del '60 la universidad pasa de ser instrumental a tener un carácter científico. Políticas institucionales como la figura del profesor investigador, la integración de las funciones sustantivas de la universidad: academia, investigación y vinculación con la sociedad, la categorización docente y la generación de tiempos exclusivos, provocan un giro estructural: la Educación Superior en el continente americano vuelca su misión al campo investigativo y al desarrollo científico. Proceso que transcurre en un escenario de tensiones económicas y políticas que ponen al descubierto la crisis del continente (Cord, 2017).

A partir de la década de los '90, el énfasis conceptual de la Educación Superior se concentra en generar los rasgos propios, el aporte específico década universidad al campo de la formación profesional. La identidad universitaria se convierte en un eje de reestructuración de las instituciones (Aboites, 2010). Países como Argentina, Bolivia y Brasil establecieron en esta década mecanismos legislativos que lograron un ordenamiento en los sistemas universitarios y la implementación del currículo por competencias (Jones, 2015). Sin embargo, en países como Ecuador, no se logró generar en esta década un cuerpo legal que condujera las reformas educativas. La universidad ecuatoriana quedó al margen de este proceso transformador (Villanueva, 2010).

El nuevo milenio trajo consigo la globalización también en el ámbito universitario. La movilidad académica y la conjunción de esfuerzos interinstitucionales en la elaboración y ejecución de proyectos integradores, produjeron giros determinantes en la Educación Superior. El aseguramiento de la calidad de la educación se convirtió en el eje de reestructuración de la universidad y las prácticas de acreditación y evaluación permitieron la legitimación académica de las instituciones. Para el contexto latinoamericano, la globalización representó también una crisis de supervivencia por su sentido excluyente. Puso en evidencia el atraso académico e investigativo de la región y marcó las brechas existentes en cuanto a uso de tecnologías, las falencias en la actualización de conocimientos y las insuficiencias en infraestructura armónica con los parámetros de calidad e inclusión. La implementación de estrategias de superación en la Educación Superior se hizo inminente como respuesta a un mundo globalizado y competitivo en condiciones de desigualdad (Knobel y Bernasconi, 2017; Silvia-Laya, 2017).

No fue sino hasta el último quinquenio que Ecuador comenzó a implementar estas prácticas en el Educación Superior del país. Esta transformación requiere necesariamente el compromiso institucional con los procesos de innovación y aseguramiento de la calidad de las tres funciones sustantivas de la universidad: academia, investigación y vinculación con la sociedad. Por supuesto que estos procesos tienen un avance paulatino que implica cambios profundos no sólo en las políticas institucionales sino, sobre todo, en la concepción de los docentes acerca de estas tres funciones y el despojo de roles y posturas obsoletas. Coincidimos con Zabalza (2012) al decir que: "No resulta difícil incorporar innovaciones a la docencia universitaria (...) Lo que parece casi inalcanzable es que esas innovaciones lleguen al currículo y sean capaces de impactar en su diseño y filosofía". Los rediseños curriculares de las carreras universitarias implican una reinterpretación de las dinámicas formativas, una nueva lectura de los paradigmas epistemológicos en la construcción del objeto de estudio, una visión pertinente del perfil de egreso, una integración de la investigación con la vinculación y una reestructuración de la organización de los aprendizajes. El currículo por competencias orienta a la docencia hacia el logro de resultados en ambientes de aprendizajes cooperativos (Larraz y Liesa, 2017; Pereira et al., 2017).

Todo esto conlleva una etapa de reconstrucción paradigmática que, en muchos casos, se ve obstaculizada por los tiempos de entrega y la burocratización del procedimiento. Al respecto, mencionan Labate (2016) y Díaz (2016) que los procesos de actualización curricular de las carreras universitarias suelen transcurrir en un marco de tensión entre los tiempos que se precisan para el debate de cátedras y con los representantes del campo profesional, que confluyen en la elaboración del perfil de egreso y la malla curricular, frente a los tiempos administrativos que demandan lograr una actualización de la carrera dentro de plazos acotados, para poder dimensionar los costos de las reformas y formalizar la comunicación a los estudiantes que deben inscribirse en la carrera renovada. 
En este contexto, el diseño de la oferta académica en Gestión Social y Desarrollo, con grado de licenciatura, responde a los parámetros de la Clasificación Internacional Normalizada de la Educación (CINE), y al Reglamento de armonización de la nomenclatura de títulos profesionales y grados académicos, expedido por el Consejo de Educación Superior (CES) de la República del Ecuador (2014). Tiene como antecedente académico la carrera denominada Ingeniería en Desarrollo Social y Cultural, cuya vigencia se ha mantenido desde el año 2002 - fecha en que fue registrada oficialmente la universidad - hasta el año 2016.

En Ecuador existen 71 universidades y escuelas politécnicas acreditadas, entre públicas y privadas (SENESCYT, 2014). De ellas, 5 ofrecen carreras de tercer nivel afines a la gestión y desarrollo. En el caso de la formación de posgrado, dos instituciones en el país presentan especialización y maestría en la misma área profesional y de ellas, una ofrece además, la formación doctoral. Debido a la poca oferta académica existente, fue necesario incorporar al estudio de tendencias académicas (Hernández, 2017), referencias universitarias de la región que enriquecieran el análisis.

Dicho estudio puso de manifiesto que países como Chile, México, Argentina y Brasil han apostado por un cambio de paradigma en cuanto a la concepción de la enseñanza superior. Al decir de Didriksson (2012), la universidad debe entenderse como una organización "de diferente nivel de participación de sus múltiples actores; flexible, auto-regulada y con una fuerte orientación de compromiso social y regional. Asimismo, con instituciones que se organizan para producir y transferir conocimientos a la sociedad de forma compleja, dinámica y diferenciada, pero interactiva y articulada".

El régimen académico debe considerar como objetivo principal la formación de competencias profesionales en correspondencia con los sistemas complejos y dinámicos que intervienen en la determinación de la pertinencia. El perfil profesional es mucho más que un cúmulo de vectores, sino que responde a una dinámica de conjunciones diversas que implican la diversificación del accionar profesional de acuerdo a la perspectiva de lo local, lo regional y lo global. Los ambientes de aprendizaje deben ser contextos de desempeño in crescendo a través de la organización de los aprendizajes, incorporando la investigación como herramienta fundamental del accionar profesional. En este sentido, la universidad ecuatoriana debe recuperar la naturaleza perdida de la academia. Al respecto, menciona Aboites (2010): Lamentablemente la teatralización de los rituales educativos es moneda corriente: el docente hace como que enseña y el estudiante hace como que aprende, es decir, todos hacemos como si la universidad tuviera sentido. Necesitamos más actores transitando por el camino de la transformación, de los cambios y proyectos, más preocupados por las instituciones en sí mismas y menos por las prebendas político-clientelares o el facilismo de ocasión.

El estudio incorporó las tendencias en el campo profesional y la demanda ocupacional de la zona de influencia, para el cual se tomaron en cuenta 1111 organizaciones sociales e instituciones relacionadas a la gestión social y el desarrollo humano, así como los graduados de la carrera de Ingeniería en Desarrollo Social y Cultural. De dicho estudio se derivó que existe una demanda ocupacional de un $67 \%$ en el área del desarrollo y la gestión social debido fundamentalmente, a que la Universidad de Otavalo se encuentra implantada en un territorio de alta diversidad cultural, desarrollo comunitario y crecimiento socio económico. Finalmente, el estudio de pertinencia incluyó el levantamiento de la demanda académica existente en la provincia, tomando en cuenta 10 unidades educativas urbanas y rurales, para un total de 2639 estudiantes encuestados. De acuerdo a los datos obtenidos, la carrera responde a un $48 \%$ de demanda académica en la zona de influencia.

El presente artículo presenta el resultado de un trabajo interdisciplinario y holístico en la construcción de un diseño curricular que puede servir de referencia metodológica para la orientación de carreras afines.

\section{METODOLOGÍA DE TRABAJO}

El análisis de los nodos críticos de la Educación Superior (Larrea, 2014), sirvió de punto de partida para la nueva conceptualización de la carrera a diseñar. Aspectos como la pertinencia del modelo educativo, la organización y evaluación de los aprendizajes, la formación y habilitación profesional del personal docente, la integración de las funciones sustantivas y la revalorización de los constructos epistemológicos, fueron las directrices para la elaboración del objeto de estudio de la carrera, la proyección del perfil de egreso, las modalidades de titulación, los lineamientos de la investigación y vinculación con la sociedad, los ejes de interculturalidad y género y la definición de los campos y disciplinas de estudio de la carrera.

La metodología desarrollada partió desde el estudio de pertinencia de la carrera en el territorio. El levantamiento de información a través de encuestas y entrevistas permitió establecer los lineamientos de construcción de la oferta académica. A partir de ello, el proceso se desarrolló mediante talleres interdisciplinarios, en siete fases secuenciales: i)definición del perfil profesional y la progresión de las 
competencias a través de la formación académica; ii) definición de los horizontes epistemológicos y construcción del objeto de estudio; iii) identificación de los campos de estudio y las disciplinas académicas; iv) precisión de objetivos y carácter de la práctica pre profesional y la investigación académica; v) determinación de las integraciones curriculares a lo largo del currículo; vi) estructuración del Plan de Estudios y mala curricular; vii) diseño de los instrumentos de aseguramiento de la calidad de la formación profesional. En la Fig.1 se muestra la secuencia de la metodología utilizada.

\section{Definición del perfil \\ profesional y la progresión de \\ las competencias a través de \\ la formación académica}
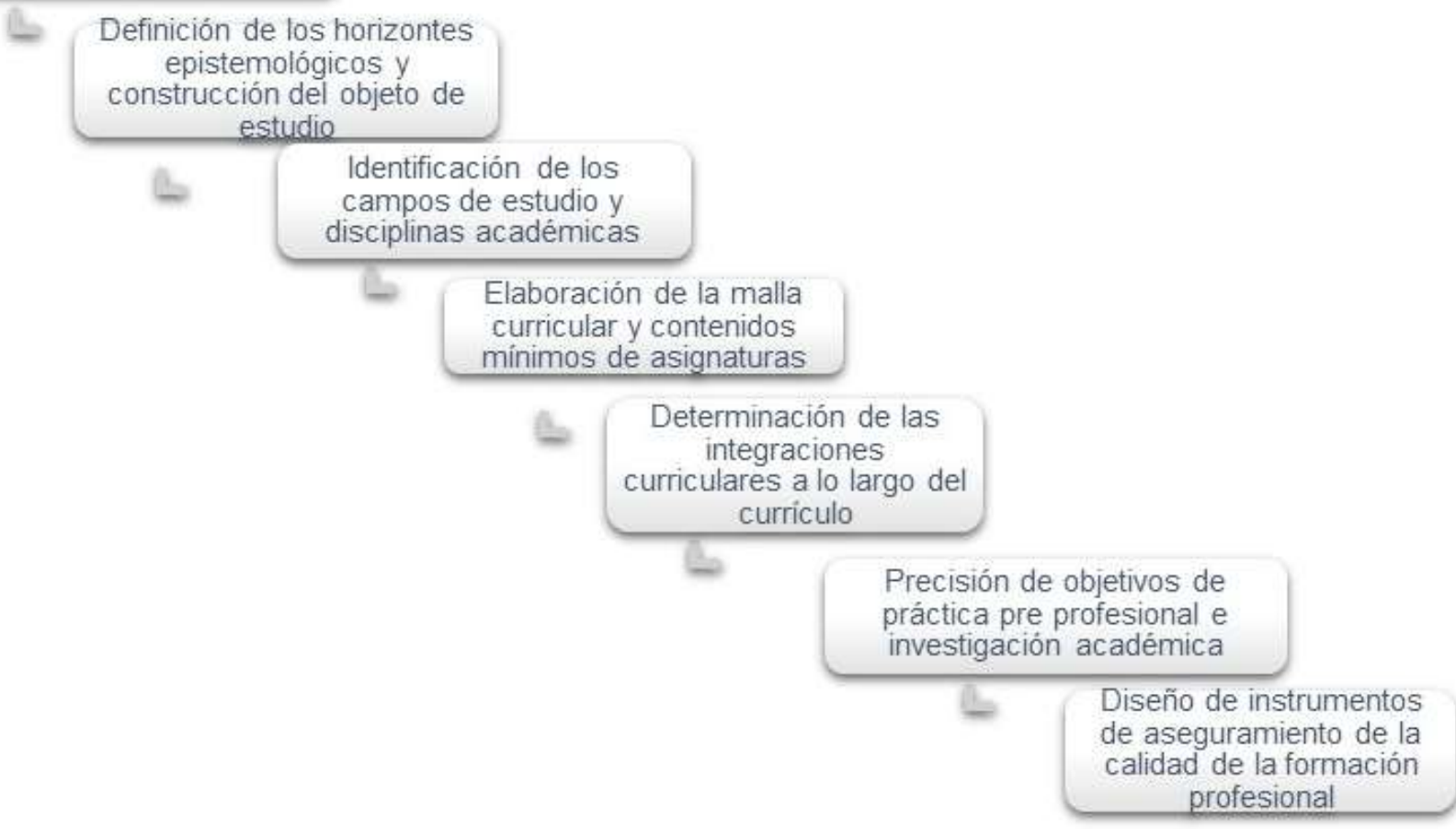

Fig.1: Diagrama de flujo de las fases de diseño curricular de la carrera Gestión Social y Desarrollo

Los talleres tuvieron un carácter participativo que incluyó a docentes, miembros del Departamento de Investigación, miembros del Departamento de Vinculación con la Sociedad, Departamento de Tecnologías Informáticas y Redes, Secretaría Académica y Biblioteca. Finalmente, una vez elaborado el diseño curricular, se procedió la validación del modelo de acuerdo con la metodología descrita por Palella y Martins (2012), de acuerdo con la cual: "La validación de modelos o aproximaciones teóricas tiene por objetivo evaluar la idoneidad de la conceptualización desde el punto de vista del procedimiento en la realidad o bien determinar su capacidad de predicción".

Para este propósito se generaron dos grupos de validación: uno con los graduados de la carrera y otro, multidisciplinar, conformado por directivos, docentes y representantes de organizaciones sociales del territorio. Los indicadores de validación utilizados, de acuerdo con los autores mencionados, fueron: Descriptivo, que explica las condiciones en que se presentan las características y condiciones del objeto de estudio; coherente, que establece las interrelaciones entre las proposiciones que la integran y aplicativo, que admite el mayor número de usos y permite explicar fenómenos. La última etapa del proceso culminó con la presentación del diseño curricular a las autoridades del Consejo de Educación Superior (CES) para su validación de expertos y posterior aprobación.

\section{PERTINENCIA DE LA GESTIÓN SOCIAL Y DESARROLLO}

El primer ámbito del análisis de pertinencia fue la articulación de la carrera con el Plan Nacional del Buen Vivir (PNBV), implementado por el Estado ecuatoriano (SENPLADES; 2013). En este sentido, se realizó un análisis multidisciplinar de los objetivos y lineamientos planteados en el PNBV y la incidencia de la gestión social en el área territorial. Dicho análisis incorporó especialistas en áreas de desarrollo local y regional, antropólogos, sociólogos y gestores culturales. De allí se determinó que el currículo aporta a los objetivos y políticas planteados en cuanto a las necesidades de desarrollo local, regional y nacional. En la Tabla 1 se exponen los objetivos y lineamientos específicos del PNBV a los cuales responde la carrera: 
Tabla 1: Objetivos y lineamientos del PNBV articulados a la carrera en Gestión Social y Desarrollo.

\begin{tabular}{|c|c|}
\hline Objetivos del PNBV & Lineamientos \\
\hline $\begin{array}{l}\text { Objetivo 2: Auspiciar la igualdad, la cohesión, la } \\
\text { inclusión y la equidad social y territorial en la } \\
\text { diversidad }\end{array}$ & $\begin{array}{l}\text { 2.11. Garantizar el BV rural y la superación de las } \\
\text { desigualdades sociales y territoriales con armonía } \\
\text { entre espacios rurales y urbanos. }\end{array}$ \\
\hline $\begin{array}{l}\text { Objetivo 4: Fortalecer las capacidades y } \\
\text { potencialidades de la ciudadanía. }\end{array}$ & $\begin{array}{l}\text { 4.8. Impulsar el diálogo intercultural como eje } \\
\text { articulador del modelo pedagógico. }\end{array}$ \\
\hline $\begin{array}{l}\text { Objetivo 5: Construir espacios de encuentro común y } \\
\text { fortalecer la identidad nacional, las identidades } \\
\text { diversas, la plurinacionalidad y la interculturalidad. }\end{array}$ & $\begin{array}{l}\text { 5.1. Promover la democratización del espacio } \\
\text { público para la construcción de relaciones } \\
\text { sociales diversas. }\end{array}$ \\
\hline $\begin{array}{l}\text { Objetivo No.6: Consolidar la transformación de la } \\
\text { justicia y fortalecer la seguridad integral, en estricto } \\
\text { respeto a los derechos }\end{array}$ & $\begin{array}{l}\text { 6.8. Promover una cultura social de paz y la } \\
\text { convivencia ciudadana en la diversidad. }\end{array}$ \\
\hline
\end{tabular}

La distinción de los objetivos del PNBV condujo al diagnóstico de las necesidades profesionales existentes en el territorio en cuanto a gestión social y desarrollo humano. Para ello se entrevistaron a los representantes de organizaciones sociales urbanas y rurales de la provincia de Imbabura. Las principales expectativas en cuanto al aporte profesional de la carrera fueron seleccionadas en el siguiente orden: planes parroquiales para el desarrollo (1); diseño e implementación de proyectos sociales (2); asesoramiento para la implementación de políticas públicas (3); fortalecimiento del estado popular de derechos (4); mejoramiento de la seguridad ciudadana (5); estrategias de economía popular y solidaria (6); defensa de los derechos colectivos (7); capacitación en democracia participativa (8); mejoramiento de las condiciones de equidad e inclusión (9); gestión de servicios y espacio público(10); mejoramiento de las condiciones de educación, salud y cultura (11); promoción de actividades culturales y deportivas (12); asesoría técnico - profesional en conflictos de frontera y gestión territorial(13). En la Fig. 2 se muestran los porcentajes alcanzados para cada uno de los ítems:

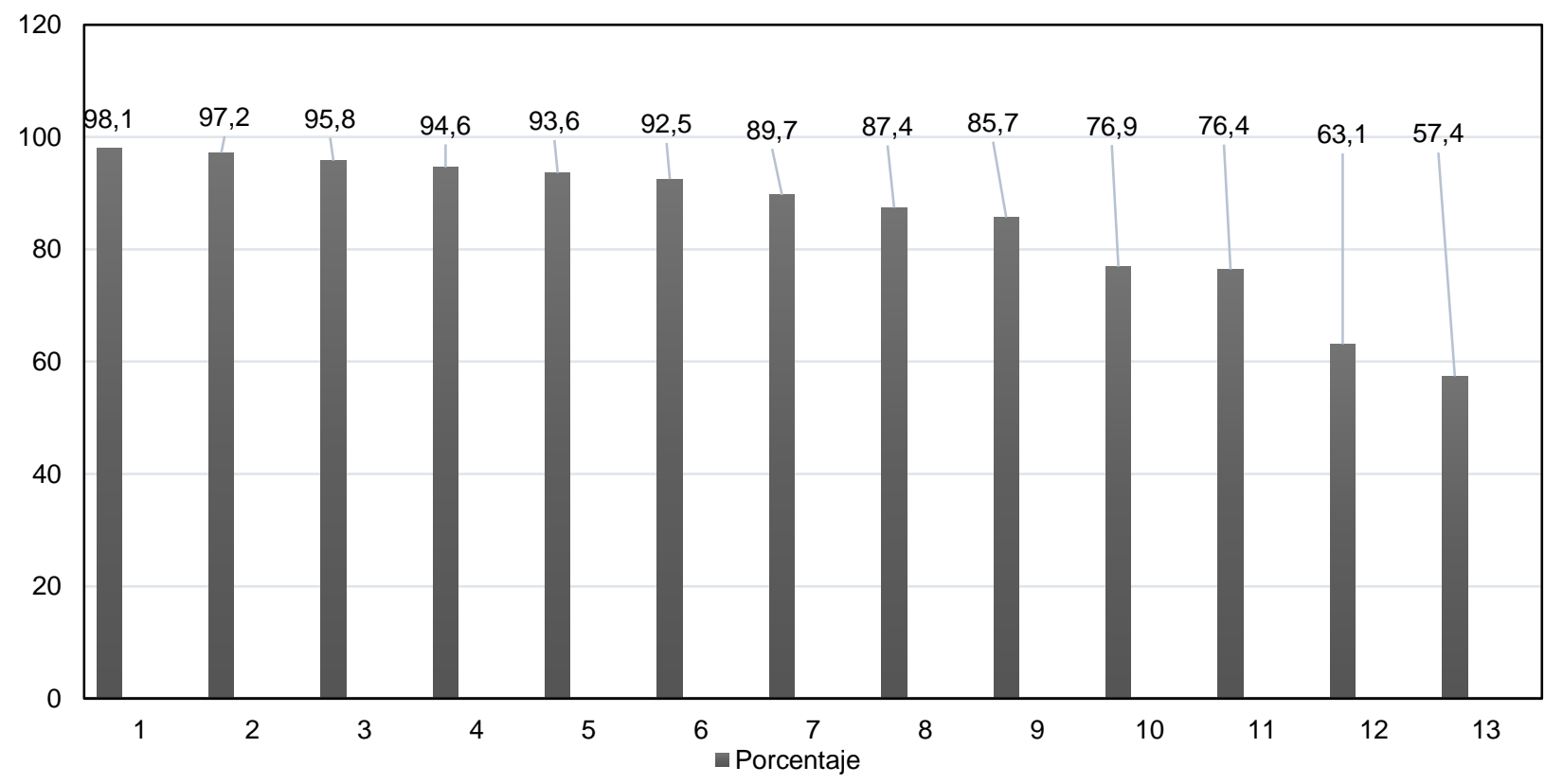

Fig. 2: Porcentajes de las competencias profesionales de la Gestión Social y Desarrollo en el territorio.

Las tendencias del desarrollo regional y local están caracterizadas por una visión armónica sobre la relación hombre - naturaleza, por las necesidades de revalorización cultural e interculturalidad, y la decolonización del conocimiento (Pirbhai - Illich y Martin, 2017). Estas tendencias son: el desarrollo humano sostenible, la gestión social enfocada en el desarrollo humano, y la gestión de las organizaciones sociales. En la Tabla 2 se resumen las tendencias encontradas: 
Tabla 2: Tendencias del desarrollo regional y local en el territorio.

\begin{tabular}{|l|l|}
\hline \multicolumn{1}{|c|}{ Tendencia } & \multicolumn{1}{c|}{ Definición } \\
\hline $\begin{array}{l}\text { Desarrollo humano sostenible enfocado hacia la calidad } \\
\text { de vida }\end{array}$ & $\begin{array}{l}\text { El desarrollo humano entendido como el crecimiento } \\
\text { progresivo de las capacidades de las personas como } \\
\text { consecuencia de la ampliación de sus oportunidades, no } \\
\text { sólo en aspectos de salud y educación, sino abarcando } \\
\text { otras dimensiones esenciales del ser social, como el } \\
\text { ejercicio de las libertades civiles y la participación en la } \\
\text { toma de decisiones para el mejoramiento de su calidad de } \\
\text { vida. En cuanto a la sostenibilidad, significa un desarrollo } \\
\text { que responde a las necesidades de la humanidad } \\
\text { presente sin comprometer la existencia de las futuras } \\
\text { generaciones. Esto significa comprender la sostenibilidad } \\
\text { como biomímesis, con formas de economías cíclicas, } \\
\text { renovables y no residuales. }\end{array}$ \\
\hline $\begin{array}{l}\text { Gestión social enfocada al acompañamiento de los } \\
\text { actores }\end{array}$ & $\begin{array}{l}\text { La gestión social desde la comprensión del tejido social } \\
\text { en su complejidad dinámica de factores políticos, } \\
\text { económicos, culturales y ambientales y como herramienta } \\
\text { de articulación sistémica. }\end{array}$ \\
\hline $\begin{array}{l}\text { Gestión de las organizaciones sociales desde la } \\
\text { perspectiva de sostenibilidad }\end{array}$ & $\begin{array}{l}\text { Una gestión que conduce al desarrollo organizacional en } \\
\text { vinculación con el contexto, a través de la sistematización } \\
\text { de procesos de diagnóstico, diseño, planeación, ejecución } \\
\text { y control de los programas y proyectos. }\end{array}$ \\
\hline
\end{tabular}

El estudio de pertinencia permitió generar la primera fase del diseño curricular: la definición del perfil profesional y la progresión de las competencias a través de la formación académica.

\section{PERFIL PROFESIONAL DE LA GESTIÓN SOCIAL Y DESARROLLO}

Partir de las competencias profesionales para el diseño curricular permite concentrar los esfuerzos disciplinares en la consecución de las capacidades profesionales de los futuros egresados. Proporciona una estrategia de evaluación del currículo, un marco de referencia para el ejercicio docente y una herramienta útil para estrechar lazos con el campo laboral (Ludvinssong, 2017; Albo, 2017; CINDA, 2008; Hawes y Corvalán, 2005). En este sentido, los autores coinciden en afirmar que el perfil profesional tiene como rol orientar la construcción del currículo, sustentar las decisiones que se tomen y ser un referente para el permanente diálogo entre los esfuerzos formadores institucionales, el mundo del trabajo y los propios practicantes de la profesión. De este diálogo surgirán orientaciones para permitir el permanente reajuste de los planes de formación e, incluso, de definiciones de competencias consideradas claves para las profesiones.

Para la definición de las competencias profesionales de la carrera, se determinaron los escenarios laborales, funciones y roles a desempeñar en el campo laboral. Coincidimos con Maura (2006) y Monedero (2013), al plantear que: la competencia profesional se manifiesta en la actuación, en tanto es en la actuación profesional que se expresan los conocimientos, hábitos, habilidades, motivos, valores, sentimientos que de forma integrada regulan la actuación del sujeto en la búsqueda de soluciones a los problemas profesionales. En la Fig. 3 se muestra el mapa conceptual de la construcción del perfil de egreso:

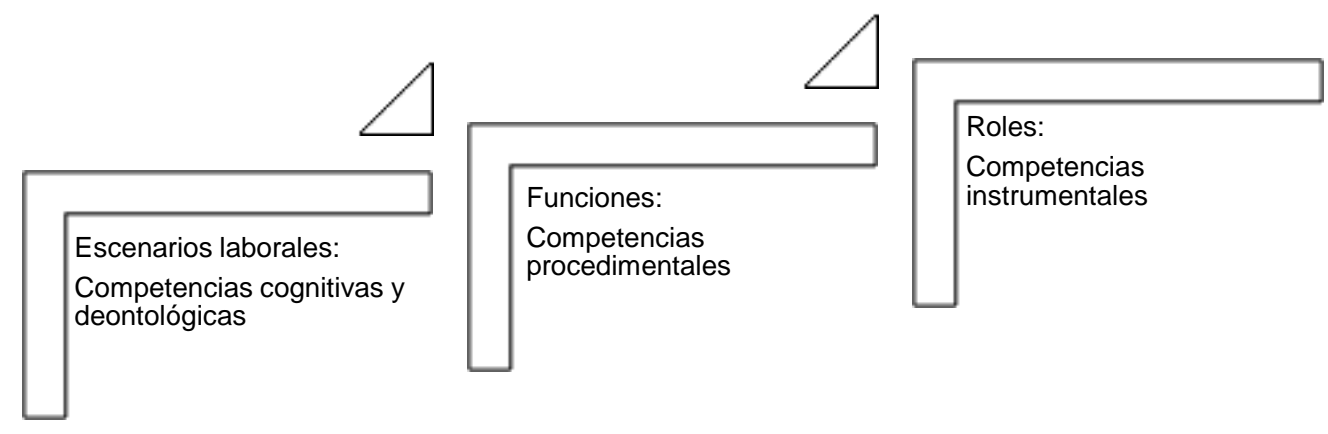

Fig. 3: Definición de las competencias profesionales a partir del perfil de egreso 
El siguiente proceso dentro de esta fase, fue establecer las etapas de progresión de las competencias profesionales en la organización de los aprendizajes. En la Fig. 4 se presenta el diagrama de dicha progresión:
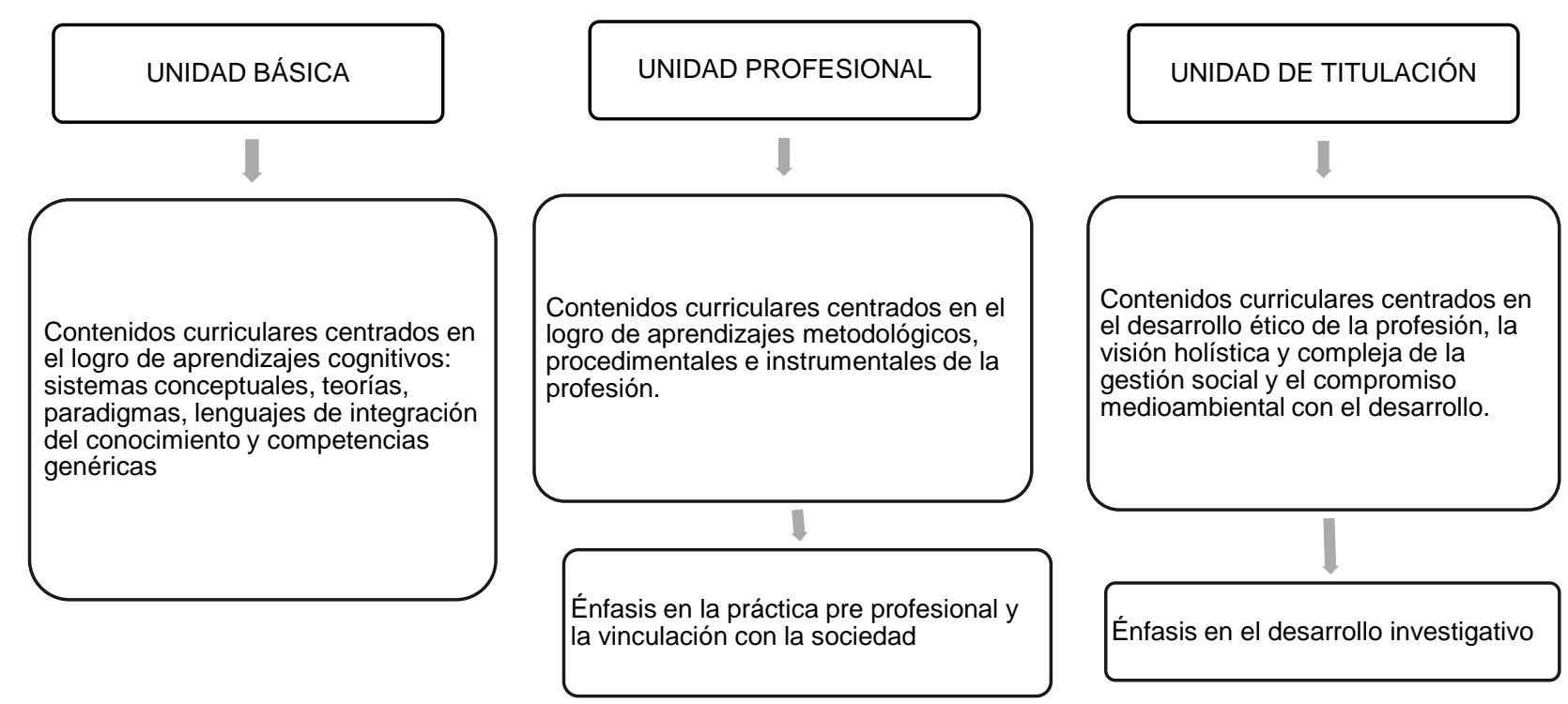

Fig. 4: Progresión de las competencias profesionales en la organización de los aprendizajes

De esta manera, en la Unidad Básica, que comprende los tres primeros períodos de formación académica los resultados de aprendizajes estarán orientados a la formación de competencias genéricas y cognitivas en relación a los sistemas conceptuales y epistemológicos que fundamentan la profesión. En este sentido, el currículo requiere la estructuración de fundamentos teóricos sólidos y un eje de praxis complementario, tal como se menciona en McMahon (2013).

La Unidad Profesional, que comprende los cuatro períodos siguientes de formación académica, permitirá la preparación del futuro egresado en las áreas metodológicas y procedimentales del ejercicio, a través del manejo de modelos, protocolos y herramientas de apoyo para la gestión social. La práctica pre profesional será el escenario ideal para la simulación el diseño, elaboración y evaluación de proyectos que afiancen las competencias instrumentales. Finalmente, en la Unidad de Titulación, que comprende los dos últimos períodos de formación académica, se integrarán las competencias cognitivas y las procedimentales a través de la formación deontológica y la investigación. Los contenidos curriculares integrarán la perspectiva de lo local, lo regional y lo global en los análisis sistémicos de los contextos.

\section{HORIZONTES EPISTEMOLÓGICOS Y OBJETO DE ESTUDIO DE LA GESTIÓN SOCIAL}

La definición de los horizontes epistemológicos propicia la gestión curricular que se centra en la organización del conocimiento. Resulta un reto renovar el ámbito educativo en el cual el estudiante es el sujeto principal del acto cognoscente en el aprendizaje vinculado a las tendencias del mundo globalizado, pero, sin olvidar los elementos particulares, culturales y locales. Para Peralvo (2016): "Los fundamentos adquieren un proceso de contextualización, de forma integral entre el conocimiento, saberes, desarrollo tecnológico, profesional, actores y sectores vinculados al desarrollo de la profesión".

Los horizontes epistemológicos presentes en la profesión se desprenden del contexto global, sin dejar de lado las experiencias locales. Para ello, resultan fundamentales como marco generalizador de la profesión los siguientes: el horizonte del enfoque socio - crítico, el horizonte del pensamiento complejo, el horizonte decontructivista, y el Paradigma Abya-yala. En la Tabla 3 se resumen sus principios fundamentales.

En la conjunción de los horizontes epistemológicos, se construye el objeto de estudio de la Gestión Social y Desarrollo, que constituye el constructo medular del currículo. La carrera estudia el tejido social en su complejidad, destacando las interacciones de los sujetos sociales y la dinámica del contexto social, económico, político, cultural y ambiental, como marco para la gestión de procesos de desarrollo humano sostenible orientados al mejoramiento de la calidad de vida y la consecución del Buen Vivir de la sociedad. Una vez definido el objeto de estudio de la profesión, se determinaron los campos de estudio, las disciplinas académicas y sus núcleos básicos. 
Tabla 3: Horizontes epistemológicos que sustentan la formación profesional de la gestión social

\begin{tabular}{|c|c|}
\hline \multicolumn{2}{|r|}{ Horizontes epistemológicos de la Gestión Social y Desarrollo } \\
\hline $\begin{array}{l}\text { Enfoque socio - } \\
\text { crítico }\end{array}$ & $\begin{array}{l}\text { Tiene como fin la emancipación y liberación de los colectivos para el cambio y la transformación aspecto } \\
\text { epistemológico principal es la búsqueda de consensos dentro de la subjetividad colectiva aplicando una } \\
\text { metodología dialógica que articula las voces que recogen la memoria histórica para sistematizar y } \\
\text { visibilizar sus contenidos desde una perspectiva de inclusión y convivencia (Maldonado, 2015). }\end{array}$ \\
\hline $\begin{array}{l}\text { Enfoque del } \\
\text { pensamiento } \\
\text { complejo }\end{array}$ & $\begin{array}{l}\text { De acuerdo con la teoría desarrollada por Morín, la incorporación del sujeto a las ciencias y las } \\
\text { ciencias a los sujetos, ha traído consigo la convergencia entre lo objetivo y lo subjetivo, provocando el } \\
\text { replanteamiento de la racionalidad occidental y generando el paradigma de la complejidad, que tiene } \\
\text { su construcción en la praxis. La complejidad intrínseca de los fenómenos y procesos biológicos, } \\
\text { físicos y sociales supera la linealidad y valida el sistema holístico y multidimensional como constructo } \\
\text { explicativo de la nueva ciencia y asegura un marco conceptual que permite establecer interrelaciones } \\
\text { entre las diversas disciplinas (Barberouse, 2008; Paiwa 2004). }\end{array}$ \\
\hline $\begin{array}{l}\text { El horizonte } \\
\text { decontructivista }\end{array}$ & $\begin{array}{l}\text { La construcción del conocimiento no puede darse exenta de la transformación permanente del } \\
\text { pensamiento a la vez que conserva el bagaje cultural e histórico que lo constituye. Esto supone la } \\
\text { incorporación dinámica de los saberes, conocimientos teóricos y aprendizajes metodológicos de } \\
\text { manera dialéctica en el lenguaje científico y filosófico. El decontructivismo produce una ruptura } \\
\text { epistemológica a la hermenéutica de las humanidades, gracias al ejercicio de detectar "lo otro" en los } \\
\text { discursos y analizar las estructuras sedimentadas que forman el elemento discursivo (Larrea, 2014). }\end{array}$ \\
\hline $\begin{array}{l}\text { El Paradigma } \\
\text { Abya-yala }\end{array}$ & $\begin{array}{l}\text { La construcción del conocimiento desde este paradigma implica la formación de un pensamiento poli } \\
\text { - lógico en el cual el ser es nudo de relaciones vitales en las cuales el "somos" ocupa la esencia } \\
\text { generadora del saber. Para (Calderón, 2014), la idea de una unidad diversa, sustentada en una } \\
\text { perspectiva múltiple, permite unas lecturas complejas, unas interrelaciones variadas, una riqueza } \\
\text { inagotable en la base misma del paradigma Abya Yala. Se trata de tensiones vinculares y solidarias } \\
\text { complejas, desplegadas e implicadas de las más diversas maneras. Múltiples ciclos, análogos pero } \\
\text { no idénticos ni antitéticos, son repeticiones cíclicas transformativas. }\end{array}$ \\
\hline
\end{tabular}

\section{CARÁCTER Y OBJETIVOS DE LA PRÁCTICA PRE PROFESIONAL}

Hasta la reciente reforma universitaria, la práctica pre profesional en la educación universitaria ecuatoriana era considerada como un elemento aislado a la formación académica. De manera desarticulada al currículo, había perdido su función formativa, convirtiéndose en un mero requisito de graduación y, en el mejor de los casos, una yuxtaposición a ciertas materias aleatorias. Una visión integrada del currículo convierte a la práctica pre profesional en eje formativo del perfil de egreso y, por ende, se articula al ámbito académico y al investigativo. Al decir de Zabalza (2011), el practicum es una pieza relevante del proceso de formación de nuestros estudiantes destinado a enriquecer la formación complementando los aprendizajes académicos (teóricos y prácticos) con la experiencia (también formativa, es decir, vinculada a aprendizajes) en centros de trabajo. De acuerdo con lo planteado, el diseño de la carrera en Gestión Social y Desarrollo integra la práctica pre profesional al currículo a través de cátedras integradoras, acordes a la progresión de las competencias profesionales. Con el objetivo de brindar a la formación académica los escenarios idóneos para desarrollar competencias genéricas, procedimentales e instrumentales, con la finalidad de transferir un conocimiento integral al futuro profesional.

Según el Reglamento del Régimen Académico, las prácticas pre profesionales deben cubrir un mínimo de 400 horas distribuidas en 160 horas de vinculación con la sociedad y 240 horas de práctica pre profesional. Los proyectos de vinculación con la sociedad han sido concebidos bajo la modalidad de convenio interinstitucional y bajo la base de un estudio diagnóstico. Se encuentran integrados a los proyectos de investigación y tienen como objetivo fundamental la integración de la universidad con la sociedad a través de estrategias de servicio a la comunidad. En la Tabla 4 se muestran las modalidades y escenarios de la práctica pre profesionales:

Tabla 4: Modalidades y escenarios de la práctica pre profesional

\begin{tabular}{|l|l|l|}
\hline Unidad de organización curricular & \multicolumn{1}{|c|}{ Modalidad } & \multicolumn{1}{|c|}{ Escenario } \\
\hline Básica & $\begin{array}{l}\text { Vinculación con } \\
\text { la colectividad }\end{array}$ & Contexto comunitario (sectores urbanos marginales y/o rurales). \\
\hline Profesional & $\begin{array}{l}\text { En el lugar } \\
\text { (in situ) }\end{array}$ & $\begin{array}{l}\text { Entidades públicas, comunidades y organizaciones sociales del } \\
\text { entorno que requieran el aporte de las competencias } \\
\text { profesionales de la Gestión Social y Desarrollo. }\end{array}$ \\
\hline Titulación & $\begin{array}{l}\text { En el lugar } \\
\text { (in situ) }\end{array}$ & $\begin{array}{l}\text { Entidades públicas, comunidades y organizaciones sociales del } \\
\text { entorno que requieran el aporte de las competencias } \\
\text { profesionales de la Gestión Social y Desarrollo. }\end{array}$ \\
\hline
\end{tabular}


Consecuentemente con esto, los objetivos de las prácticas estarán en relación con las competencias instrumentales que pueden ser desarrolladas en estos escenarios. En la Tabla 5 se expone la relación entre dichas competencias y los objetivos planteados. Para ello, la práctica pre profesional se llevará a cabo a través de las siguientes cátedras integradoras: Desarrollo Social y Planificación; Gestión Social; Proyectos Sociales; Gerencia organizacional; Desarrollo Comunitario Local y Regional. A través del desarrollo de los proyectos integradores, estas asignaturas permitirán la formación de las competencias profesionales necesarias para la actuación como gestores sociales.

Tabla 5: Relación entre las competencias específicas y los objetivos de la práctica pre profesional

\begin{tabular}{|c|c|c|}
\hline $\begin{array}{l}\text { Unidad de } \\
\text { organización } \\
\text { curricular }\end{array}$ & $\begin{array}{c}\text { Competencias específicas a desarrollarse en la } \\
\text { práctica }\end{array}$ & Objetivo de la práctica \\
\hline Básica & $\begin{array}{l}\text { Conocimiento de la estructura organizacional y } \\
\text { procedimental de instituciones y organizaciones } \\
\text { que trabajen por el desarrollo humano sostenible. } \\
\text { ldentificación de la normativa y estrategias para la } \\
\text { implementación de políticas públicas. } \\
\text { Manejo de las estrategias de formulación e } \\
\text { implementación de programas y proyectos de } \\
\text { gestión institucional, comunitaria y } \\
\text { emprendimientos sociales. }\end{array}$ & $\begin{array}{l}\text { Comprender las líneas y estrategias de la } \\
\text { planificación social para el desarrollo. }\end{array}$ \\
\hline Profesional & $\begin{array}{l}\text { Diseño, ejecución y evaluación de proyectos } \\
\text { sociales } \\
\text { Uso de la logística operativa en la articulación con } \\
\text { los actores sociales. } \\
\text { Manejo de herramientas socioeducativas. } \\
\text { Mapeo de actores. } \\
\text { Capacidad para el análisis situacional. }\end{array}$ & $\begin{array}{l}\text { Aplicación de la metodología de la Gestión } \\
\text { Social a escenarios concretos. } \\
\text { Aplicación de las fases y ciclo de los } \\
\text { proyectos en el diseño y elaboración de los } \\
\text { mismos. } \\
\text { Aplicar la socio educación en escenarios } \\
\text { reales. } \\
\text { Realizar mapeo de actores sociales. } \\
\text { Analizar las áreas estratégicas de la } \\
\text { Gestión Social en el territorio. }\end{array}$ \\
\hline Titulación & $\begin{array}{l}\text { Capacidad para reconocer las dinámicas } \\
\text { culturales que integran el tejido social. } \\
\text { Capacidad para adaptar estrategias de gestión } \\
\text { social a la realidad concreta de los contextos. }\end{array}$ & $\begin{array}{l}\text { Identificar las áreas, procesos y } \\
\text { dimensiones del desarrollo comunitario, } \\
\text { local y regional, desde la perspectiva de la } \\
\text { inclusión intercultural y el respeto a los } \\
\text { Derechos Humanos y la participación } \\
\text { social. }\end{array}$ \\
\hline
\end{tabular}

\section{CARÁCTER Y OBJETIVOS DE LA INVESTIGACIÓN}

La investigación, en el marco de la transformación de la docencia universitaria ecuatoriana, alcanza su carácter formativo, inter y multidisciplinar a través de los proyectos integradores. Estos imprimen la flexibilidad que requiere el currículo para responder a las necesidades del futuro profesional. Orientada desde la perspectiva de las competencias profesionales, la investigación en la Gestión Social se convierte en eje transversal para la intervención en contextos donde lo social, lo cultural, lo económico, lo político, lo ambiental, lo local y lo global confluyen de manera particular dentro de cada sistema complejo. El tejido social es el campo de transformación de la investigación. Concordamos con Bravo, Illescas y Lara (2017) en que se investiga para transformar la realidad y "con ello contribuir al desarrollo humano y por lo tanto mejorar la calidad de vida, por lo que ella se constituye en un medio muy valioso para lograr cualquier transformación en el ámbito profesional".

En este sentido, la investigación formativa conduce al estudiante a una postura crítica sobre la realidad. El desarrollo de habilidades metodológicas conceptuales superiores permite que la información se convierta en conocimiento real, apropiándose de manera consciente de las operaciones estructurales que procuran la adquisición de dicho conocimiento. Los proyectos integradores, desde la metodología del método científico y el aprendizaje basado en problemas (ABP), ofrecen múltiples posibilidades de acción práctica y valorativa que van más allá de la aplicación del propio método. Estas metodologías son, precisamente, las generadoras de las habilidades metacognitivas. 
Los proyectos integradores permiten la participación activa del estudiante en el proceso investigativo y generan la cultura de resolución de problemas desde el marco de la investigación - acción participativa. De acuerdo con Fong et al., (2016), los proyectos integradores involucran a todas, o a casi todas las asignaturas de un nivel de estudio, para solucionar un problema profesional real, ya sea social, tecnológico o empresarial realizado en cada uno de los niveles de estudio de una carrera. Resulta medular para el profesional en Gestión Social y Desarrollo la formación en las habilidades de investigación. El modelo de investigación de la carrera responde al paradigma de la investigación - acción participativa que converge con la modalidad de los proyectos integradores. A lo largo del currículo las cátedras integradoras son las herramientas pedagógicas ideales para el desarrollo de las competencias que permitirán a los futuros profesionales desempeñarse eficazmente en una sociedad plural, comunicarse y relacionarse con los demás, valorar las diferencias, expresar las propias ideas, cooperar, ser empático, comprometerse, tomar decisiones, reflexionar, resolver conflictos, ser tolerante y respetar los valores, las creencias, las culturas y la historia personal y colectiva.

Una vez definidos los objetivos y carácter de la investigación y la práctica pre profesional, se determinaron las integraciones curriculares a través de las cuales se incorporan esto aspecto de la formación académica. La elaboración de la malla curricular, a partir de los campos de estudio y el diseño de los contenidos mínimos de las asignaturas tuvo un total de seis talleres interdisciplinares.

\section{ESTRUCTURA DEL PLAN DE ESTUDIOS Y MALLA CURRICULAR}

Con los elementos anteriores, se procedió a estructurar el plan de estudios y elaborar la malla curricular. Tomando en cuenta las orientaciones dadas por el Reglamento del Régimen Académico (RRA, 2014) y documentos afines (Larrea, 2014), el plan de estudios se estructuró de acuerdo a la normativa planteada por el Consejo de Educación Superior (CES), con una organización del aprendizaje en nueve períodos académicos, tres unidades de formación y en una relación de 1:1,5 entre las horas de docencia y horas de trabajo autónomo (CES 2014). Para Vargas (2011), el plan de estudios es una "descripción general de lo que será aprendido por el estudiante y cuánto tiempo requiere para ello. Su función es la de informar a profesores, educandos y administradores sobre lo que ha de aprenderse y el orden que seguirá en el proceso". Elaborar el plan de estudios implica tres actividades: seleccionar los contenidos, derivar de los objetivos generales los objetivos específicos a partir de los cuales se estructurarán los cursos y desglosar los que formaran parte del plan de estudios. De esta manera, los contenidos curriculares se definieron de acuerdo a los objetivos generales del plan y se establecieron los procedimientos e instrumentos de evaluación. En la Fig. 5 se muestra el proceso de elaboración seguido:

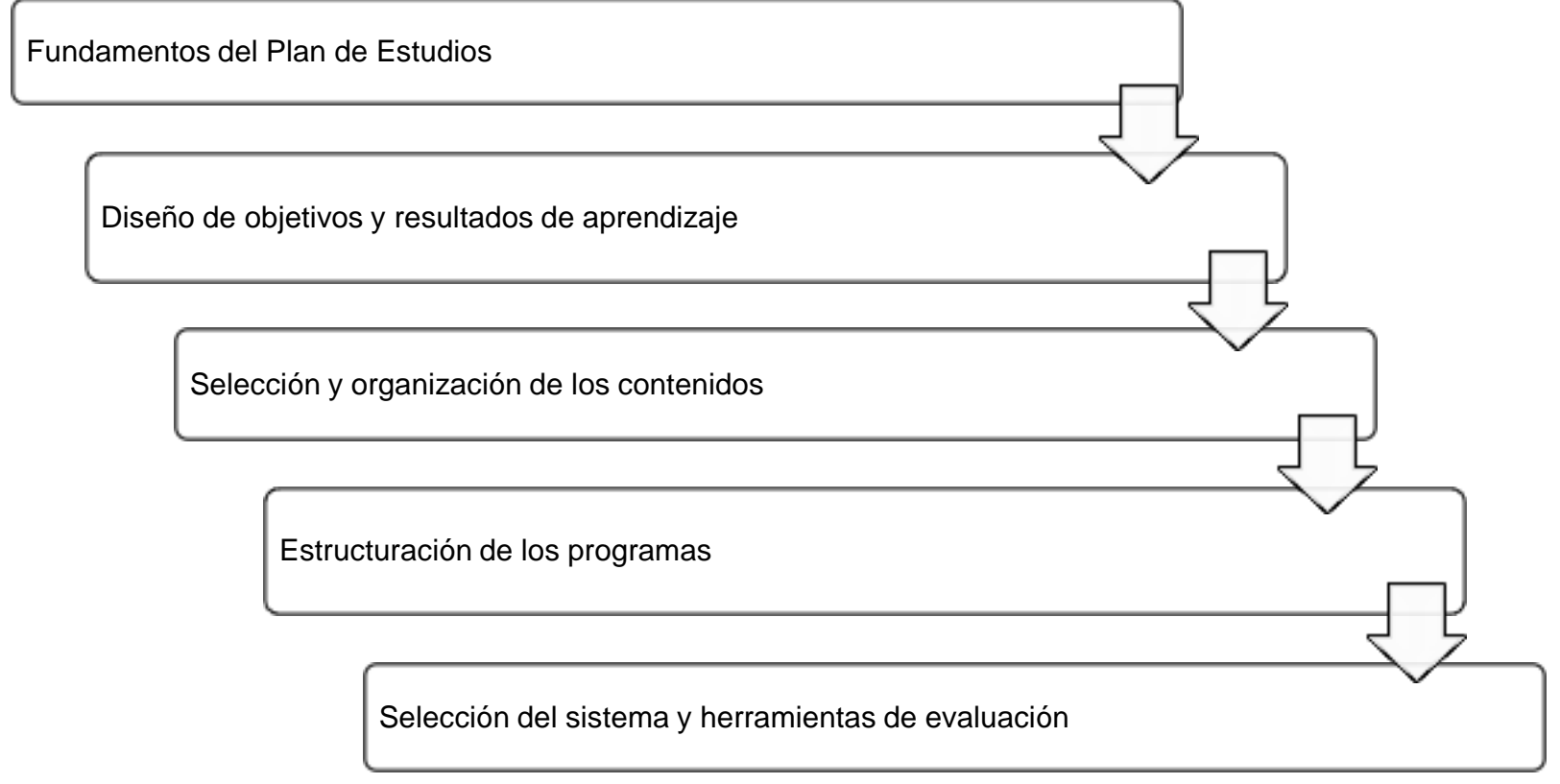

Fig. 5: Diagrama del proceso de diseño del plan de estudios de Gestión Social y Desarrollo

La elaboración de la malla curricular partió del análisis de los campos de estudios, siguiendo los cinco ejes de formación establecidos por el RRA (Larrea, 2014), siendo estos: i) eje de fundamentos teóricos; ii) eje de praxis profesional; iii) eje de Epistemología y Metodología de la Investigación; iv) eje de integración de saberes, contextos y culturas; v) eje de comunicación y lenguajes. 
A través de dichos ejes se estructuran los campos de estudio y las disciplinas curriculares, conforme a la progresión de las competencias profesionales y estableciendo las interrelaciones curriculares y las cátedras que regirán los proyectos integradores. El campo de estudio medular de la formación profesional es la gestión social, la cual rige el comportamiento de la malla curricular. Como ejes transversales del conocimiento se incluyeron la interculturalidad y el género, expresados en los contenidos curriculares y resultados de aprendizaje de las asignaturas. La delimitación de los campos de estudios fue realizada de acuerdo a la visión de Zabalza (2012), para quien el currículo debe ser concebido como un proyecto formativo integrado, buscando establecer nexos entre las disciplinas y tratando de generar una coreografía didáctica con el propósito de alcanzar el aprendizaje significativo de los estudiantes.

En síntesis, la malla curricular fue conformada en una extensión de 9 niveles, 7200 horas y 51 asignaturas relacionadas en una proporción de 1 a 1,5 entre las horas de docencia y las de aprendizaje autónomo. Se describen cuatro campos de estudio: ciencias básicas, ciencias disciplinares, ciencias instrumentales e investigación dentro de los cuales se organizan todas las disciplinas académicas. Hasta el séptimo nivel, se imparten 6 asignaturas por semestre. Los semestres octavo y noveno, correspondientes a la Unidad de Titulación, que tienen cinco asignaturas cada uno, incorporando más horas de docencia al trabajo de titulación.

Dentro de las ciencias básicas, se incluyen asignaturas correspondientes a las disciplinas: Sociología, Antropología Sociocultural, Filosofía, Política, Ecología y Economía. Dentro de las ciencias disciplinares, se incluyen asignaturas correspondientes a las disciplinas: Gestión Social y Desarrollo Humano. Dentro de las ciencias instrumentales se incluyen asignaturas correspondientes a las disciplinas: Psicología Social, Planificación Estratégica y Tecnologías de la Información y Comunicación. En el campo de estudio de la investigación se incluyen las asignaturas relacionadas a la metodología de la investigación, haciendo énfasis en la investigación social. Las cátedras integradoras tienen la función de conducir el aprendizaje de los estudiantes incorporando los saberes de varias disciplinas en la ejecución de proyectos de investigación y vinculación. Estas asignaturas están directamente relacionadas a la formación práctica del estudiante, por lo que corresponden a la disciplina de la Gestión Social.

\section{ASEGURAMIENTO DE LA CALIDAD EN LA FORMACIÒN PROFESIONAL}

Asegurar la calidad en la formación profesional se convierte en la estrategia idónea para la evaluación integral del currículo. En ella están inmersos los procesos pedagógicos de enseñanza -aprendizaje que garantizan los resultados de aprendizaje y formación de competencias profesionales. Los ámbitos de aseguramiento de la calidad se encuentran en estrecha relación con las funciones sustantivas de la universidad: i) academia; ii) investigación y iii) práctica pre profesional y vinculación con la sociedad. En la Fig. 6 se presenta la relación entre dichos ámbitos.

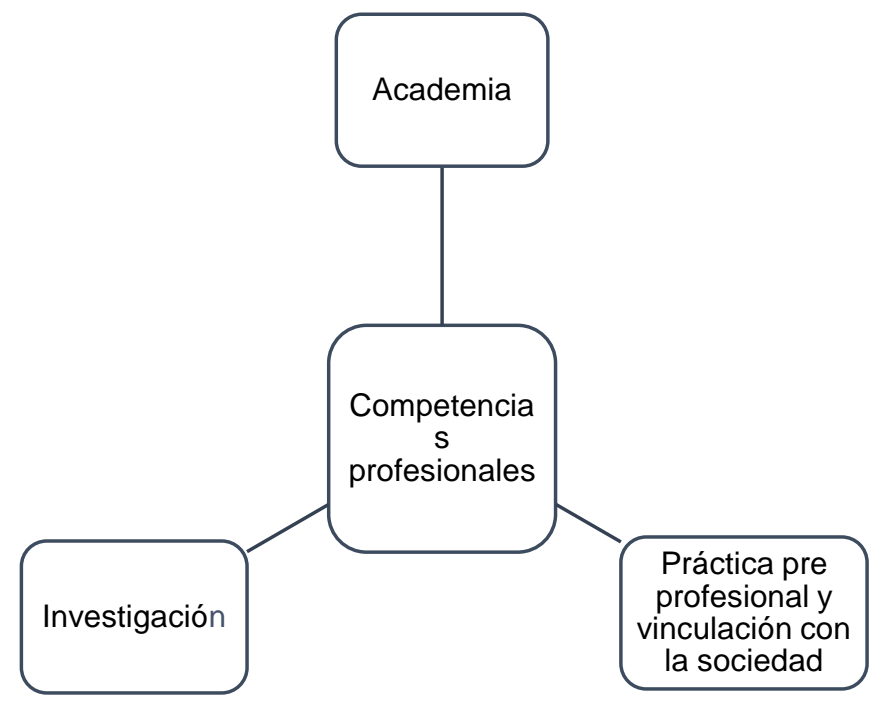

Fig. 6: Relación entre los ámbitos de aseguramiento de la calidad en la formación profesional.

La metodología e instrumentos diseñados para la evaluación de dicha calidad responden a los objetivos de cada uno de los ámbitos. Para el ámbito academia se diseñaron tres instrumentos: el examen de resultados de aprendizajes, el proyecto de investigación y el examen complexivo final. Para la práctica pre profesional, la vinculación con la sociedad y la investigación, la metodología planteada es a través de los resultados de 
los proyectos integradores, mediante los siguientes instrumentos de evaluación: el informe del proyecto integrador: Informe escrito por los estudiantes que reúne los aspectos metodológicos y resultados alcanzados en el proyecto integrador; el informe del tutor del proyecto, que constituye una evaluación integral del tutor, que expone no sólo los resultados del proyecto integrador sino además las actitudes y desempeños del estudiante durante el desarrollo del mismo; y el aval de la organización externa.

A lo anterior se añade el análisis integral de la pertinencia del currículo, la evaluación integral docente y la actualización de contenidos mínimos de las asignaturas. Estos procedimientos serán llevados a cabo con una frecuencia de dos años y mediante la adaptación de metodologías descritas en la literatura (Mata, 2015; Maldonado, 2016; Mandujano y Morales, 2016; Díaz, 2016). Para dicho análisis se siguen los parámetros e indicadores establecidos en el modelo para la evaluación de carreras presenciales y semi presenciales del Consejo de Aseguramiento y Acreditación de la Calidad de la Educación Superior (CEAACES).

\section{CONCLUSIONES}

El presente diseño curricular resulta una innovación respecto a la concepción de la formación profesional en Gestión Social y Desarrollo para la Universidad de Otavalo. La visión holística de las problemáticas existentes, la conjunción con las políticas macro del país, el currículo por competencias profesionales, la investigación y la práctica pre profesional como componentes estructurales de la formación académica, la composición de integraciones curriculares, muestran un avance significativo para la institución en su oferta académica.

La metodología utilizada permite el enfoque integral y multidisciplinario de los nodos críticos que originan la necesidad del análisis del currículo y la conducción del diseño. El estudio de pertinencia ofrece el panorama contextual desde las tendencias académicas y profesionales, que constituye la base para la demanda del currículo. La definición de los horizontes epistemológicos proporciona el marco referencial filosófico para la construcción del objeto de estudio, los campos de estudio, las disciplinas académicas y los núcleos básicos de formación.

La determinación del modelo de investigación y el modelo de práctica pre profesional enmarca las acciones específicas de la formación en las competencias relacionadas al "saber hacer" del ejercicio profesional. El diseño de procedimientos y herramientas para el aseguramiento de la calidad en la formación profesional orienta los mecanismos de análisis progresivo del currículo, garantizando su flexibilidad y evolución.

El presente diseño curricular debe ser sometido a un proceso de acreditación periódico, de acuerdo a la normativa, para garantizar la progresión de su calidad y los reajustes requeridos por los nuevos enfoques y reformas de la Educación Superior. La inestabilidad existente en los procesos de redes académicas entre las universidades del país que ofrecen carreras afines, sin dudas, es una falencia en la construcción dinámica de los currículos universitarios.

\section{REFERENCIAS}

Aboites, H.; Higher education in Latin America and the Bologna process: from commercialization to the adoption of the tunning competition project, https://goo.gl/MF5eXC, ISSN: 0798-1228 15, Las Transformaciones de la Educación Superior en América: Identidades en Formación (1) 27-43 (2010)

Albo, M.V.; Tunning history in Latin America, doi: 10.1177/1474022216686522, Arts and Humanities in Higher Education, 16(2) 14-28 (2017)

Barberouse, P.; Fundamentos teóricos del pensamiento complejo de Edgar Morín, https://goo.gl/EJxmjj, ISSN: 1409-42-58, Educare, XII (2), 95-113 (2008)

Bravo, G.; S. Illescas y L.M. Lara; El desarrollo de las habilidades de investigación en los estudiantes universitarios, una necesidad para la formación de investigadores, https://goo.gl/gGG9vU, ISSN: 2308-1953, Revista de Educación, Cooperación y Bienestar Social, 10, 23-32, p. 24 (2017)

Calderón, M.; La construcción de una propia matriz civilizatoria como "ruta" en la búsqueda del Buen Vivir, doi: http://dx.doi.org/10.15359/siwo.7-2.4, Siwo 7(2), 2-7 (2014)

Centro Universitario de Desarrollo (CINDA), Grupo operativo de universidades chilenas, Fondo de desarrollo institucional; Diseño curricular basado en competencias y aseguramiento de la calidad en la Educación Superior, 1를 Ed., 2-509, MINEDUC, Santiago de Chile (2008) 
CINE: Organización de las Naciones Unidas para la Educación, la Ciencia y la Cultura (UNESCO), Clasificación Internacional Normalizada de la Educación, París, Francia (2016)

Cord, L.E.; Inequality Stagnation in Latin America in the Aftermath of the Global Financial Crisis, doi: 10.1111/rode.12260, Review of Development Economics, 21(1), 157-181 (2017)

Díaz, M.B.; Curriculum Management and the Role of Curriculum Actors, https://goo.gl/qwh7YR, ISSN: 14498855, Transnational curriculum inquirí, The journal of international association for the advacemento of curriculum studies, 13 (2) 13-33 (2016)

Didriksson, A.; The new transformation agenda in Latinamerica higher education, https://goo.gl/PzJW5y, ISSN: 0185-2698, Perfiles Educativos, 34(138), 184-203, p.195 (2012)

Fong, W.; R. Acevedo y C. Severiche; Estrategia de investigación formativa tecnológica: el caso del proyecto integrador, ISSN: 0121-2753, Itineraria Educativo, 67, 103-121 (2016)

Hawes, G. y O. Corvalán; Construcción de un perfil profesional, Universidad de Talca, Instituto de Educación y Desarrollo Profesional (2005)

Hernández, L.; Estudio de tendencias en la formación profesional de la carrera gestión social y desarrollo, https://goo.gl/8sytvX, ISSN: 1390-9207, Revista Sarance, 37, 47-61 (2017)

ITESO, Universidad Jesuita de Guadalajara, Cátedra para la gestión social y el desarrollo humano sostenible, http://www.iteso.mx/catedras/unesco (2015)

Jones, G.; Competence an undestanding - a personal perspective, en Key competences in physics teaching and learning, 1를. Ed., Ewa, 3-9, Wroclaw, Poland (2015)

Knobel, M. y A. Bernasconi; Latin American universities: stuck in the twentieth century, International Higher Education, doi: 10.6017/ihe.2017.88.9693, 88, 26-28 (2017)

Labate, G.A.; Metodología para la revisión y actualización de un diseño curricular de una carrera universitaria incorporando conceptos de aprendizaje basado en competencias, doi:10.4067/S071850062016000200002, Revista Formación Universitaria, 9(2), 3-16, p.3 (2016)

Larraz, N. y S.V. Liesa; Transversal skills development through cooperative learning. Training teachers for the future, https://goo.gl/b1AuYh, ISSN: 1074-8121, On the horizon, 25, 16-31 (2017)

Larrea, E., Unidad curricular de titulación. Consejo de Educación Superior, Ecuador (2014)

Larrea, E.; El currículo de la educación superior desde la complejidad sistémica, Consejo de Educación Superior, Ecuador (2014)

LOES: Secretaría de Educación Superior, Ciencia y Tecnología (Ecuador), Ley Orgánica de Educación Superior, Quito (2011)

Ludvinssong, A.B.; Tunning history, doi: 10.1177/1474022216686507. Arts \& Humanities in Higher Education, 1-4 (2017)

Maldonado, A.; Paradigma sociocrítico en la investigación social, 1ㅡ Ed., 2-78, Paidós, Buenos Aires, (2015)

Maldonado, L.C.; Evaluación de competencias docentes en el desarrollo de la práctica pedagógica, doi:199502ZU2628, Omnia, 22(2), 37-49 (2016)

Mandujano, L. y V.G. Morales; Evaluación curricular e idoneidad docente, ISSN: 2448-6574, Debates en Evaluación y Currículum. Memorias del Congreso Internacional de Educación, 2(2), 3422-3430 (2016)

Mata, Y.E.; Procedimiento para la evaluación de la pertinencia interna y externa de un currículo en Venezuela, doi::201502DC4596, Educación en Contexto, 1(1), 57-77 (2015)

Maura, V.G.; La formación de competencias profesionales en la universidad. Reflexiones y Experiencias desde una perspectiva educativa, https://goo.gl/sL1YP6, ISSN: 1575-0345, Rev. de Educación, XXI, 8, 175-187, p. 179 (2006) 
McMahon, J.P.; Applied genre analysis: the case of introductions in spanish teacher trainees curricular design documents, Doctorado, Universidad Complutense de Madrid (2013)

Monedero, J. C.; Curso urgente de política para gente decente, 1를 Ed., 1-248, Seix Barral, Madrid, España (2013)

Paiwa, A., Edgar Morin y el pensamiento de la complejidad, https://goo.gl/djxjzY, ISSN: 0210-9581, Ciencias de la Educación,1 (23), 239-253 (2004)

Palella, S. y S.P. Martins; Metodología de la investigación cuantitativa, 3aㅡ Ed., 19-275, p.33, FEDUPEL, Caracas, Venezuela (2012)

Peralvo, C.; Bases y fundamentos del currículo genérico de las carreras de Ciencias de la educación del Ecuador, https://goo.gl/96hMB1, ISSN: 2224-2643. Didasc@lia: Didáctica y Educación, 83-90, p. 88 (2016)

Pereira, A., R.A. Giménez y R.G. Tusing; Implementation of competency-based curriculum: College of Philosophy, doi: 10.1002/cbe2.1038, The Journal of competency- base education, (1), 1-9 (2017)

Pirbhai-Illich, F. y S.P. Martin; Culturally Responsive Pedagogy: Decolonization, Indigeneity and Interculturalism, 1a Ed., 12-67, Springer International Publishing AG, Bern, Switzerland (2017)

RRA: Consejo de Educación Superior (Ecuador), Reglamento del Régimen Académico, Quito (2014)

RTPGA: Consejo de Educación Superior (Ecuador), Reglamento de armonización de la nomenclatura de títulos profesionales y grados académicos que confieren las Instituciones de Educación Superior del Ecuador, Quito (2014)

Secretaría Nacional de Planificación y Desarrollo (Senplades), Agenda zonal para el desarrollo. Zona de planificación 1. 1aㅡ Ed., Senplades, Quito, Ecuador (2013)

Secretaría Nacional de Planificación y Desarrollo (Senplades), Plan Nacional del Buen Vivir (PNBV), $1^{\underline{a}}$ Ed., 13-594, Senplades, Quito, Ecuador (2013)

Silva-Laya, C.; Higher education for sustainable development at EARTH University, doi:10.1108/IJSHE-062015-0104, International Journal of Sustainability in Higher Education, 18 (3), 278 - 293 (2017)

Vargas, J. A.; Planeación educativa y diseño curricular: un ejercicio de sistematización, Boletín Electrónico de Investigación de la Asociación Oaxaqueña de Psicología A.C., 7(1), 53-64, p. 56 (2011)

Velasco, J. M.; El bucle educativo: aprendizaje, pensamiento complejo y transdisciplinareidad, Modelos de planificaciones aula metacomplejos, https://goo.gl/ZjnQQV, Íntegra Educativa, 2 (2), 83-101 (2012)

Villanueva, E.; Perspectivas de la Educación Superior en América Latina: construyendo futuros, https://goo.gl/cwMs1W, ISSN: 0185-2698, Perfiles educativos, 33(129), 86-101 (2010)

Zabalza, M.; Articulación y rediseño curricular: el eterno desafío institucional, ISSN: 1887-4592, Revista de Docencia Universitaria REDU, 17-48 (2012)

Zabalza, M.; El practicum en la formación universitaria: estado de la cuestión, https://goo.gl/uq7CVn, ISSN: 034-8082, Revista de Educación, 254, 21-47, pp.18, 25 (2011) 